\title{
El estudio contrastivo-pragmático entre primeras y segundas lenguas de los registros formal y coloquial
}

\author{
The contrastive-pragmatic study of formal \\ and colloquial registers \\ between first and second languages
}

\author{
Raúl Fernández Jódar \\ Uniwersytet im. Adama Mickiewicza w Poznaniu (Polonia) \\ raulfdez@amu.edu.pl
}

\begin{abstract}
In this article we intend to present the theoretical bases for the contrastive study of colloquial and formal registers from a pragmatic point of view. Although we start from the point of view of contrastive linguistics, we consider that neither the comparison of the theoretical grammars of two languages, nor the description of specific actions of learners of an L2, can show the nuances of the formal and colloquial registers.

Formal and colloquial registers should not be understood as watertight departments with no relationship between them, but as two extremes within the continuum of speech. Obviously, this continuum will differ depending on the language in question, since certain lexical, pragmatic or syntactic elements, among others, can be understood as corresponding to different registers.

Now, how should we face the contrast of this continuum belonging to different languages? In the paper we present both the techniques used to measure the attitude of the interviewees regarding different elements of the colloquial and formal records, as well as the concepts that will help us to analyse the data obtained. Finally, the analysis can draw conclusions that refer to the learning process and the teaching process.
\end{abstract}

Keywords: register, colloquial, formal, Spanish as a foreign language, pragmatics, transfer, pragmatic error 


\section{INTRODUCCIÓN}

Todo profesor de idiomas, así como cualquier persona que haya tenido que comunicarse en una lengua extranjera o con un interlocutor cuya lengua materna fuera diferente a la propia, es consciente de que dominar las cuatro destrezas no es siempre sinónimo de una comunicación efectiva. En ocasiones, la comunicación puede resultar fallida, a pesar de que aparentemente nuestro interlocutor tiene un nivel de conocimiento de la lengua elevado, ya sea porque nos puede resultar demasiado directo o indirecto en sus intervenciones (Fernández Jódar, 2003) ${ }^{1}$ o no se recurre al uso de las formas lingüísticas pertinentes para cada función comunicativa y registro. En estos casos el problema suele residir en una competencia pragmática que ha sido complementada por la lengua materna del aprendiz y que difiere de la lengua meta.

Es evidente que la lengua materna (L1) funciona en ocasiones como estrategia de aprendizaje al ser la fuente de la que se obtienen los conocimientos que se desconocen de la lengua meta y en otras como estrategia de comunicación al producir textos orales o escritos. Ahora bien, mientras que la transferencia negativa de aspectos puramente lingüísticos como el uso de tiempos verbales, formas verbales, pronombres, preposiciones, etc. suele ser reconocida como error por el interlocutor, la transferencia negativa pragmática puede ser causa de conflictos comunicativos. Así, un hablante de segunda lengua (L2) puede parecernos impertinente o maleducado al usar determinado vocabulario en contextos inadecuados sin que fuera su voluntad. Sin embargo, tampoco debemos caer en la falsa creencia de que todos los errores vienen causados por una interferencia de la L1. En ocasiones, la transferencia de práctica o instrucción también puede ser el origen de una competencia pragmática insuficiente.

Nuestra intención en el presente artículo es presentar una metodología de análisis contrastivo-pragmático que pueda dilucidar hasta qué punto se produce una transferencia de las características de los registros formal y coloquial entre primeras y segundas lenguas, así como cuáles pueden ser las causas de dichos trasvases. De esta manera, se podrán entender mejor los errores de tipo pragmalingüístico (Fernández Jódar, 2015).

\section{LA PRAGMÁTICA EN LOS ESTUDIOS SOBRE TRANSFERENCIA}

Los estudios por transferencia en los procesos de adquisición de lenguas segundas se iniciaron en base a cuatro niveles como son el fonético-fonológico, el morfológico, el sintáctico y el léxico-semántico. Sin embargo, estos niveles no daban cuenta de ciertos errores que no podían ser clasificados y eran descritos como actitudes poco naturales o extrañas por parte de aprendices de L2.

\footnotetext{
${ }^{1}$ En dicho artículo ya mostramos cómo una de las posibles causas de incomprensión entre hablantes de diferentes lenguas maternas podía ser la organización del discurso y la forma directa o indirecta de mostrar las tesis defendidas por parte del hablante.
} 
Gracias a los estudios pioneros de Austin y su teoría de los actos de habla (1962), de Searle (1969) o de Grice (1989) y al auge a partir del último cuarto del siglo XX de la Pragmática, el Análisis del discurso o la Etnografía se inició el estudio de la transferencia pragmática. Cabe añadir que el concepto de competencia comunicativa (Hymes, 1971; Canale, Swain, 1980; Llobera, 1995) acentuó la idea de que un hablante no requiere solamente de conocimientos lingüísticos para comunicarse plenamente en una L2, sino también de conocimientos pragmáticos, es decir, se comprendió que esas actitudes poco naturales eran una muestra de discrepancia cultural aplicada a la L2. A partir de ahí, surgieron numerosos estudios sobre cortesía, peticiones, disculpas, invitaciones, etc. En estos trabajos se suelen comparar los elementos lingüísticos que aparecen en los actos de habla de diferentes lenguas con la intención de una posterior aplicación en el aula de idiomas, mostrando las faltas de equivalencias que son causa de errores pragmáticos.

En general, se suele señalar que la transferencia negativa pragmática se debe a la aplicación de normas de la L1 a la L2 como estrategia de comunicación sin tener en cuenta si estas normas son equivalentes. En realidad, como veremos en puntos posteriores, no toda transferencia pragmática es una estrategia de comunicación ni siempre es la L1 el origen de estas discrepancias.

\section{EL REGISTRO Y SU DIFICULTAD DE ANÁLISIS}

El estudio de los registros, especialmente del registro coloquial, ha superado cualquier prejuicio que pudiera haber, convirtiéndose en materia de estudio con pleno derecho. Es bien conocida la aportación de Briz Gómez, fundador e impulsor del Grupo Val.Es.Co., junto con las publicaciones de Vigara (1992), Hidalgo (1997) Ruiz Gurillo (1998) o Gaviño Rodríguez (2008), entre otros.

En estos trabajos se trata el registro coloquial como un extremo opuesto al registro formal, pero con una frontera difusa entre ambos. Entre dichos extremos nos encontramos con una serie de características más propias de un registro o del otro. Como afirma Briz Gómez (1996) los registros formal e informal " "podrían ser entendidos como dos extremos imaginarios dentro del continuum del habla, extremos no entendidos tanto como límites a uno y otro lado del continuum, sino como cortes más o menos arbitrarios en el mismo" (p. 16). En ocasiones un discurso puede presentar aspectos propios del registro formal entremezclados con otros más coloquiales, siendo la suma de todas estas características lo que nos hará percibir un discurso como más o menos formal o coloquial. Es decir, la frontera entre ambos registros no se puede marcar con una línea.

\footnotetext{
${ }^{2}$ Compartimos con Gaviño Rodríguez (2008) la idea de que el español coloquial se sitúa en la zona del registro informal al oponerse a los "estilos planificados que están en la órbita de la modalidad formal" (p. 18), por lo que ambos términos funcionarían como sinónimos.
} 
Fig. 1. Eje de los registros (Elaboración propia)

Sin embargo, no todas las tradiciones se enfrentan al estudio de los registros de la misma forma. En la lingüística polaca, L1 de los aprendices de español con los que trabajamos, el debate va por otros derroteros, ya que se busca una definición de lo coloquial que presente claramente el objeto de estudio, de ahí que sea más frecuente que en la bibliografía especializada se trate más el tema de qué significa coloquial y no una descripción gramatical stricto sensu. Así, los autores de referencia de los registros en polaco, como Anusewicz (1992), Barmiński (1991) o Hołówka (1986), entre otros, suelen hacer hincapié en definiciones basadas en un supuesto sentido común y pragmático que nos hace percibir "lo coloquial" como un estilo o variante-el debate terminológico continúa- de la lengua oficial, utilizado en el día a día, en determinados contextos y por personas concretas, principalmente jóvenes. De esta forma, parece darse a entender que lo coloquial no puede aparecer más allá de unas fronteras claramente delimitadas por categorías estilístico-situacionales. Una excepción sería la obra de Zdunkiewicz-Jedynak (2008), quien en sus trabajos presenta las características gramaticales propias del registro coloquial frente al registro formal.

Así, si la descripción de los registros en una lengua puede resultar complicada, contrastar los registros de dos lenguas cuyas tradiciones parten de descripciones diferentes, resultará más arduo si cabe. Por eso, un análisis contrastivo-pragmático que pretenda dejar al descubierto errores de carácter pragmalingüístico no debería partir de la descripción teórica de dos lenguas, sino de datos obtenidos de aprendices de una L2 en contraste con datos obtenidos de hablantes que tengan dicha L2 como L1. Si bien esta idea parece aceptada desde hace años en la lingüística contrastiva -lo que dio como resultado el análisis de errores y la descripción de las diferentes interlenguas ${ }^{3}$-, en los estudios de pragmática todavía continúa dándose.

Ahora bien, tampoco es adecuado partir de la comparación de actos de habla reales en diferentes lenguas. Como veremos en el siguiente apartado, los datos analizados no corresponderán a producciones reales e intentaremos ir un paso más lejos en la descripción de los registros en una interlengua mediante el estudio de nuevos parámetros.

${ }^{3}$ En el caso del español y del polaco disponemos de una descripción de la interlengua por parte de aprendices polacos de español (Fernández Jódar, 2006), así como de la Gramática contrastiva español-polaco (Nowikow, 2017). 
Es decir, consideramos que la descripción de los diferentes actos de habla puede ser necesaria para la creación de material de una L2. Sin embargo, contrastar elementos lingüísticos que aparecen en actos de habla de diferentes lenguas solo mostrará las divergencias, las cuales han sido normalmente descritas como causantes de transferencias pragmáticas negativas aplicadas en estrategias de comunicación. De esta manera, se obvia que pueden existir otras causas como la transferencia de instrucción.

\section{METODOLOGÍA}

En los siguientes apartados presentamos los parámetros que debemos tener en cuenta para llevar a cabo un estudio contrastivo-pragmático y el proceso de obtención de estos.

\subsection{PARÁMETROS}

Tomemos en consideración las palabras galleta, torta y mamporro (Fernández Jódar, 2020), las cuales aparecen recogidas por el DLE como sinónimas de bofetada (s.f., s.v. bofetada) y descritas como coloquiales. Un usuario de la lengua no solo conocerá su significado por el contexto en el que aparezcan, sino que también las reconocerá como propias del registro coloquial. A un usuario de una L2 generalmente se le exige lo mismo, que disponga de determinado vocabulario y, en función del nivel, sepa reconocerlo como coloquial o formal.

Ahora bien, un hablante nativo también dispone de una percepción más amplia con la que es capaz de asignar un diferente grado de coloquialidad a cada uno de los tres términos. De esta manera, podrá dilucidar incluso si en un contexto formal podría aparecer alguno de ellos y situaría las tres palabras sinónimas de bofetada en diferentes puntos en el continuum de los registros, posiblemente considerando mamporro como las más coloquial de ellas.

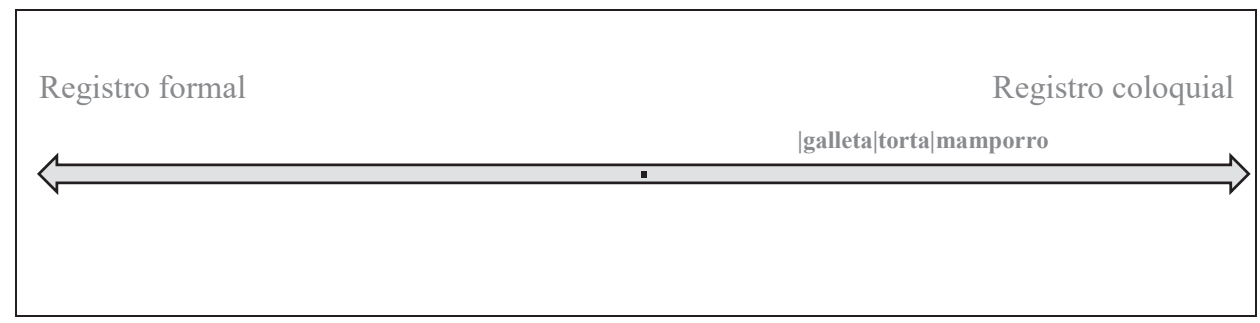

Fig. 2. Sinónimos de bofetada en el eje de los registros (Elaboración propia) 
Esta percepción no es inherente solamente a los hablantes de una L1. Sin embargo, para los hablantes de una L2 es más difícil de adquirir, puesto que no forma parte explícita de los manuales, ni en los diccionarios se formula de forma precisa; por ello, se necesitan contextos reales fuera del aula para poder adquirirla. En el caso de que esto no ocurra, la percepción que se aplique será la transferida de la L1 o la adquirida por transferencia de instrucción.

Otro ejemplo (Fernández Jódar, 2019c) serían las locuciones verbales que el DLE presenta como sinónimas agachar el lomo (s.f., s.v. lomo) y romperse alguien los cuernos (s.f., s.v. cuerno). Dichas locuciones, aunque en el DLE se describan como coloquiales, no se percibirán con un mismo grado de coloquialidad por parte de los hablantes.

Un último ejemplo sería el verbo dar, el cual en el DLE es descrito como coloquial en solo dos de sus 53 acepciones (s.f., s.v. dar) y en algunas locuciones. Según se deduce de esto, dar un beso y besar según el DLE se situarían en un mismo punto en el eje de los registros. Sin embargo, para los hablantes nativos, según comprobamos en Fernández Jódar (2020), un pro-verbo se percibe generalmente con un mayor grado de coloquialidad. Es decir, hay características del registro coloquial como los pro-verbos que ni siquiera aparecen descritos como tales en el DLE.

Vistos los ejemplos anteriores, parece claro que los parámetros con los que nos vamos a manejar son el conocimiento, el reconocimiento y la percepción, los cuales nos permitirán responder a las siguientes preguntas:

1. ¿Conoce el aprendiz el significado de un determinado recurso de la lengua? (conocimiento)

2. ¿Puede el aprendiz determinar si dicho recurso es propio del registro formal o del coloquial? (reconocimiento)

3. ¿Hasta qué punto puede el aprendiz precisar si un recurso es más o menos formal o más o menos coloquial? (percepción)

En función de la competencia lingüística de un aprendiz de L2, será capaz de responder a la primera, a la primera y segunda o a la totalidad de las preguntas. Asimismo, a medida que se avanza en los procesos de enseñanza se debería poder responder a dichas preguntas.

\subsection{INFORMANTES}

Evidentemente, para tratar el conocimiento, el reconocimiento y la percepción como parámetros, debemos convertirlos en datos. El primer paso consiste en determinar las características de los sujetos de estudio, los cuales estarán divididos en por lo menos dos grupos:

- Informantes aprendices de L2. Deben poseer un mismo nivel de conocimiento de la L2 acreditado por la pertenencia a un mismo grupo académico. De esta forma, también se puede evaluar de manera más fiable la transferencia de instrucción. 
Sin embargo, otras cuestiones que pueden resultar de interés son el género, el conocimiento de otras lenguas o estancias puntuales o prolongadas en países en los que se hable la lengua meta, ya sea por motivos personales o por una estancia Erasmus. En función de los aspectos que se quieran investigar, los informantes aprendices de L2 serán divididos en más de un grupo ${ }^{4}$.

- Informantes de L1. Recordemos que no basamos el análisis contrastivo-pragmático en descripciones teóricas, sino en parámetros como el conocimiento, el reconocimiento y la percepción. Estos deben ser también convertidos en valores numéricos en lo concerniente al grupo de hablantes con el que se realizará el contraste y que, si se prefiere, puede ser considerado como grupo de control.

Para obtener datos contrastables lo más fiables posibles, las diferencias entre ambos grupos deben ser mínimas, incluso reducirlas al único factor relevante que es la diferente L1. Por lo que el grupo de informantes de L1 debe tener el mismo nivel educativo que los informantes aprendices de L2.

Según Labov (1972), la cantidad idónea de informantes para considerar que los datos obtenidos son representativos es de un $0,025 \%$ de la población total. En realidad, ese porcentaje se podría superar con creces si los informantes seleccionados tienen una de las características anteriormente mencionadas: pertenencia a un mismo grupo académico. Así, no se analizarían los registros de toda una comunidad lingüística, sino de una comunidad de habla circunscrita a un contexto educativo de una zona determinada.

\subsection{RECOGIDA DE DATOS}

La recogida de datos se realiza mediante encuestas basadas en pruebas de evocación. En estas generalmente se solicita al hablante que evalúe la aceptabilidad o gramaticalidad de una o más construcciones gramaticales o léxicas.

Sin embargo, en nuestro caso los participantes no deben considerar la aceptabilidad o gramaticalidad de diferentes elementos, sino puntuar en una escala que va de 5 a -5 palabras, sintagmas, breves diálogos o breves diálogos con elementos destacados, presentados en listados. El 5 representaría el grado máximo de formalidad y el -5 el grado máximo de coloquialidad. Consideraremos los resultados entre $-0,5$ y 0,5 como equivalentes a un valor neutro. Los elementos desconocidos no deben puntuarse. De esta manera se puede evaluar el conocimiento de los diferentes elementos, los cuales representan características léxicas, sintácticas o conversacionales propias de los registros.

${ }^{4}$ En Fernández Jódar (2019b, 2019b y 2020) los datos se obtuvieron de dos grupos de informantes aprendices polacos de L2 divididos en función de si habían estado acogidos al programa Erasmus o nunca habían realizado una estancia superior a dos semanas en un país de habla española. 
En estas encuestas los elementos deben aparecer sin contexto ni ninguna explicación que pueda modificar la percepción que los sujetos tienen de ellos. La intención no es evaluar la influencia del contexto en determinado elemento, ni el contexto en sí mismo, sino el reconocimiento y la percepción que tiene el informante de dicho elemento. Recordemos que no analizamos actos de habla, sino los registros, los cuales aparecen transversalmente en todos los actos de habla. De esta manera, no nos centramos en los contextos en los que se utilizarían las palabras galleta, torta o mamporro vistas anteriormente, sino en el valor que el informante les otorga en sí mismas.

\section{CONCLUSIÓN}

Es indiscutible la necesidad de avanzar en los estudios contrastivo-pragmáticos para dilucidar la influencia de los procesos de transferencia, del tipo que sea, en la enseñanza y el aprendizaje de L2, puesto que a pesar del interés y de los avances sigue siendo un campo en el que quedan grandes zonas por investigar.

Consideramos que la metodología propuesta puede ayudar en este avance. Así, por ejemplo, nos ha posibilitado visibilizar cómo los pro-verbos son percibidos como neutros o formales entre los aprendices de L2 debido a la transferencia de instrucción (Fernández Jódar, 2019b), la dificultad del conocimiento y reconocimiento de las abreviaciones como creaciones léxicas en aprendices que no han realizado una estancia prolongada en un país en el que se hable la lengua meta (Fernández Jódar, 2019a) o la transferencia de la L1 en cuanto a las preferencias léxicas en una L2 (Fernández Jódar, 2020). Por otra parte, confiamos en continuar profundizando en el estudio contrastivo-pragmático entre primeras y segundas lenguas de los registros formal y coloquial, y en los procesos de transferencia pragmática derivados de este. 


\section{BIBLIOGRAFÍA}

Anusiewicz, J. (1992). Potoczność jako sposob doświadczania świata i jako postawa wobec świata. In J. Anusiewicz \& F. Nieckula (Eds.), Język a kultura, t. 5: Potoczność w języku i w kulturze (pp. 9-20). Wrocław: Wydawnictwo Uniwersytetu Wrocławskiego.

Austin, J.L. (1962). How to do things with words. Cambridge: Harvard University Press.

Bartmiński, J. (1992). Styl potoczny. In J. Anusiewicz \& F. Nieckula (Eds.), Język a kultura, t. 5: Potoczność w języku i w kulturze (pp. 37-54). Wrocław: Wydawnictwo Uniwersytetu Wrocławskiego.

Bartmiński, J. (1993). Styl potoczny. In J. Bartmiński (Ed.), Encyklopedia kultury polskiej XX wieku, t. 2 (pp. 115-134). Lublin: Wydawnictwo Wiedza o Kulturze.

Briz, A., Gómeez Molina, J.R., Martínez Alcalde, M.J., Grupo Val.Es.Co. (Eds.) (1997). Pragmática y gramática del español hablado. El español coloquial. Zaragoza: Pórtico.

Briz, A., Grupo Val.Es.Co. (2002). Corpus de conversaciones coloquiales. Madrid: Arco/Libros.

Briz Gómez, A. (1996). El español coloquial. Situación y uso. Madrid: Arco/Libros.

Canale, M., Swain, M. (1980). Theoretical Bases of Communicative Approaches to Second Language Teaching and Testing. Applied Linguistics, 1, 1-47.

Fernández Jódar, R. (2003). La organización del discurso en polaco y español como generador de incomprensión. In. A. Gregori i Gomis et al. (Eds.), Actas del Coloquio "El enfoque social y cultural en los estudios lingüísticos y literarios” (pp. 125-134), Poznań: Wydawnictwo Naukowe UAM.

Fernández Jódar, R. (2006). Análisis de errores léxicos, morfosintácticos y gráficos en la lengua escrita de los aprendices polacos de español. Tesis doctoral. Recuperado de: http://www.educacionyfp.gob. es/en/mc/redele/biblioteca-virtual/numerosanteriores/2007/memoriamaster/2-trimestre/fernandez-j. $\mathrm{html}$.

Fernández Jódar, R. (2015). El español coloquial en la clase de E/LE en contexto académico. Teoría y práctica. In Tendencias en la enseñanza de español LE. Perspectivas glotodidácticas y metodológicas contemporáneas (pp. 277-296). Kraków: Księgarnia Akademicka.

Fernández Jódar, R. (2019a). Reconocimiento y percepción de las creaciones léxicas en el registro coloquial español por parte de alumnos polacos. In A. Cabedo Nebot \& A. Hidalgo Navarro (Eds.), Pragmática del español hablado. Hacia nuevos horizontes (pp. 521-530). Valencia: Publicacions de la Universitat de València.

Fernández Jódar, R. (2019b). Sobre las preferencias léxicas en los registros coloquial y formal en L1 y L2. Studia Romanica Posnaniensia, 46/4, 49-161.

Fernández Jódar, R. (2019c). Bases para el análisis contrastivo del léxico coloquial español y polaco. In A.M. López González, M. Baran, A. Kłosińska-Nachin \& E. Kobyłecka-Piwońska (Eds.), Voces dialogantes. Estudios en homenaje al profesor Wiaczesław Nowikow (pp. 177-185). Lódz: Wydawnictwo Uniwersytetu Łódzkiego.

Fernández Jódar, R. (2020). Reconocimiento y percepción del registro coloquial español en alumnos polacos: el caso de los pro-verbos. In N. Nowikow et al. (Eds.), Lingüistica hispánica teórica y aplicada: Estudios léxico-gramaticales, didácticos y traductológicos (pp. 77-92), Lódz: Wydawnictwo Uniwersytetu Łódzkiego.

Gaviño Rodríguez, V. (2008). Español coloquial. Pragmática de lo cotidiano. Cádiz: Servicio de Publicaciones de la Universidad de Cádiz.

Grice, H.P. (1989). Studies in the way of words. Cambridge: Harvard University Press.

Gumperz, J., Hymes, D. (1972). Directions in Sociolinguistics: The Ethnography of Communication. New York: Holt, Rinehart \& Winston.

Hidalgo, A. (1997). La entonación coloquial. Función demarcativa y unidades de habla. Valencia: Publicacions de la Universitat de València. 
Hołowka T. (1986). Myślenie potoczne. Heterogeniczność zdrowego rozsadku. Warszawa: Państwowy Instytut Wydawniczy.

Hymes, D. (1971). On communicative competence. Philadelphia: University of Pennsylvania Press.

Labov, W. (1972). Language in the Inner City: Studies in the Black English Vernacular. Philadelphia: University of Pennsylvania Press.

Llobera, M. et al. (1995). Competencia comunicativa. Documentos básicos en la enseñanza de lenguas extranjeras. Madrid: Edelsa.

Nowikow, W. (2017). Gramática contrastiva español-polaco. Lódz: Wydawnictwo Uniwersytetu Łódzkiego.

Ruiz Gurillo, L. (1998). La fraseología del español coloquial. Barcelona: Ariel.

Searle, J. (1969). Speech Acts. Cambridge: Cambridge University Press.

Serrano, M.J. (2011). Sociolingüística. Barcelona: Serbal.

Vigara, A.M. (1992). Morfosintaxis del español coloquial. Madrid: Gredos.

Zdunkiewicz-Jedynak, D. (2008). Wyktady ze stylistyki. Warszawa: PWN. 